\section{NSCLC: Mutationslast ist ein wichtiger Biomarker für die Immuntherapie}

Hellmann MD et al. Nivolumab plus Ipilimumab in Lung Cancer with a High Tumor Mutational Burden. N Engl J Med 2018; 378: 2093 - 2104

In einer Phase-I-Studie war die Kombination von Nivolumab und Ipilimumab zur Behandlung des nicht kleinzelligen Bronchialkarzinoms (NSCLC) wirksam. Schon in dieser Studie zeigte sich, dass die Mutationslast ein Biomarker für das Ansprechen sein könnte. Das bestätigte sich jetzt für eine Mutationslast von $\geq 10$ Mutationen pro Megabasenpaaren in einer offenen Phase-III-Studie mit Patienten mit einem Stadium-4-NSCLC oder einem NSCLC-Rezidiv.

Die nicht mit Chemotherapie vorbehandelten Patienten wurden im Verhältnis 1:1:1 in die folgenden Gruppen randomisiert:

- Nivolumab (3 mg/kg Körpergewicht [KG] alle 2 Wochen) plus Ipilimumab $(1 \mathrm{mg} / \mathrm{kg}$ alle 6 Wochen, Nivo + Ipi),

- Nivolumab-Monotherapie (Dosierung bei PD-L1 $\geq 1 \%$ : $240 \mathrm{mg}$ alle 2 Wochen, bei PD-L1<1\%: $360 \mathrm{mg}$ alle 2 Wochen, Nivo) oder

- Chemotherapie alleine (platinhaltige Dublette nach Histologie, Chemo).

Parallel wurde die Tumormutationslast nach dem FoundationOne CDx-Assay als ein möglicher Biomarker mit untersucht. Ein koprimärer Endpunkt der Studie ist das progressionsfreie Überleben (PFS).

\section{Ergebnisse}

Wie Matthiew D. Hellmann vom Memorial Sloan Kettering Cancer Center Hospital, New York, und Kollegen berichteten, war das PFS von Patienten mit einer hohen Tumorlast ( $\geq 10$ Mutationen pro Megabasenpaaren) unter der kombinierten Checkpointblockade mit Nivo+Ipi signifikant länger als mit Chemo. Die Ein-Jahres-PFS-Rate betrug 42,6\% unter Nivo+ Ipi gegenüber $13 \%$ unter Chemo, das mediane PFS in den beiden Studienarmen lag bei 7,2 Monaten (95\% Konfi- denzintervall [KI] 5,5-13,2) versus 5,5 Monaten (95\% KI 4,4-5,8). Das entspricht einer Reduktion des Risikos für Progress oder Tod von $42 \%$ (Hazard Ratio 0,58; 97,5\% KI 0,41-0,81; $\mathrm{p}<0,001)$. Die objektive Ansprechrate (ORR) lag mit 45,3\% unter Nivo + Ipi ebenfalls signifikant höher als bei Chemo (26,9\%). Das Ansprechen war zudem im kombinierten Immuntherapie-Arm bei $68 \%$ der Patienten mit Ansprechen auch über ein Jahr anhaltend, bei Chemotherapie galt das nur für $25 \%$ der so behandelten Patienten. Grad-3/4-Nebenwirkungen betrafen $31,2 \%$ im kombinierten Immuntherapie- und $36,1 \%$ im ChemoArm.

Patienten mit einer hohen Mutationslast profitierten von Nivo+Ipi unabhängig von PD-L1-Expressionsniveau oder histologischem Subtyp. Patienten mit einer niedrigeren Mutationslast profitierten dagegen nicht von der Immuntherapie es zeigten sich keine PFS-Unterschiede zwischen Nivo-Ipi und ChemotherapieArm.

FAZIT

Die Studie weist daraufhin, dass Patienten mit einer hohen Mutationslast von einer kombinierten Checkpointblockade als Erstlinientherapie profitieren können. Damit ist die Mutationslast ein wichtiger und unabhängiger Biomarker beim fortgeschrittenen NSCLC. In der Studie war allerdings mangels adäquatem Material nur bei $58 \%$ der Patienten die Mutationslast bestimmbar. In der Praxis sollte daher von Beginn an daran gedacht werden, ausreichend Material für die Mutationsanalyse zu gewinnen.

Friederike Klein, München 